\title{
Adaptación al castellano de la Centrality of Event Scale (CES)
}

\section{Adaptation of Centrality of Event Scale (CES) to Spanish}

Recibido: 9 de julio de 2013 | Revisado: 10 de marzo de 2014 | Aceptado: 5 de febrero de 2015

\author{
MANUEl FernándeZ-AlcánTARA* \\ MACARENA DE LOS SANTOS-ROIG ** \\ M Nieves PÉREZ-MARFIL *** \\ ANDRÉS CATENA-MARTÍNEZ ***** \\ Miguel PÉREZ-García ***** \\ Celia MARTÍ-GaRCÍA $* * * * * *$ \\ FRANCISCO CRUZ-QUINTANA ******** \\ Universidad de Granada, España
}

\section{RESUMEN}

La "Centrality of Event Scale" (CES) es un instrumento utilizado para evaluar la centralidad que tiene una determinada experiencia en la persona. Estudios previos muestran que la centralidad es un constructo altamente relacionado con el estrés post-traumático, la depresión o el duelo complicado. El objetivo de este estudio fue realizar su adaptación al castellano y caracterizar su fiabilidad y validez. Participaron dos muestras compuestas por 208 y 320 estudiantes universitarios que completaron la CES y medidas de depresión (BDI), ansiedad (STAI) y sintomatología de estrés post-traumático (PSS). Los resultados coinciden con los obtenidos con la versión original de la CES, tanto en consistencia interna (alfa de Cronbach de 0.92 y 0.94, para cada muestra), como en test-retest a los dos meses $(r=0.803, p<0.01)$. El análisis de componentes principales muestra un único factor explicativo que daba cuenta del $45 \%$ de la varianza. Finalmente las puntuaciones de la CES muestran relaciones con diversos índices de psicopatología, siendo además un predictor, junto con las medidas de ansiedad y depresión, del 32\% de la sintomatología de estrés post-traumático. La adaptación al castellano de la escala CES parece ser una medida válida y fiable sobre centralidad del evento. Palabras Clave

TEPT, Trauma, Memoria Autobiográfica, Identidad, Centralidad

\section{A B S TRACT}

The "Centrality of Event Scale" (CES) is an instrument used to assess the centrality that a particular event has in the person. Previous studies show that centrality is a construct highly relevant for post-traumatic stress symptomathology, depression and complicated grief. The aim of this study was to adapt into Spanish the CES and obtain evidences of reliability and validity. Two samples consisting of 208 and 320 college students participated in the study. They completed the CES and measures of depression (BDI), anxiety (STAI) and symptoms of post-traumatic stress (PSS). The results agree with those obtained by the original version of the CES, showing high internal consistency (Cronbach's alpha of 0.92 and 0.94 , for each sample) and acceptable test-retest reliability at two months $(\mathrm{r}=0.803, \mathrm{p}<0.01)$. The principal component analysis shows a single explanatory factor that accounted for $45 \%$ of the variance. Finally, CES scores show significative relationships with various indicators of psychopathology, and is also a predictor, along with measures of anxiety and depression, of the $32 \%$ of the symptoms of post-traumatic stress disorder. The Spanish version of the CES seems to be a valid and reliable measure of the centrality of the event. Keywords

PTSD, Trauma, Autobiographic Memory, Identity, Centrality 


\section{Introducción}

La memoria autobiográfica es un componente de la memoria episódica y se relaciona con el recuerdo de sucesos de la historia vital de la persona. La memoria autobiográfica requiere de una serie de capacidades psicológicas relacionadas con la percepción de un self o yo, así como de una linealidad temporal en que este pueda situarse (Klein, German, Cosmides, $\&$ Gabriel, 2004).

Hay diferentes aproximaciones que tratan de explicar la organización y codificación de los recuerdos autobiográficos, y más concretamente los relacionados con situaciones traumáticas o de gran estrés emocional. Estas últimas son cambiantes y dependientes de diversos factores, como el grado de recuperación o de resiliencia de la persona (Dekel \& Bonnano, 2013) o ciertas variables de personalidad (Moreno, Morante, Rodríguez, \& Rodríguez, 2008).

La primera aproximación asume que experiencias de sucesos potencialmente traumáticos tendrían dificultades para integrarse dentro de la memoria autobiográfica y que un déficit de integración sería un componente básico en el surgimiento y mantenimiento del trastorno de estrés postraumático (TEPT), en el que los recuerdos se encuentran fragmentados y poco integrados (Dalgleish, 2004). Así, los pacientes con estrés postraumático (Dalgleish, Rolfe, Golden, Dunn, \& Barnard, 2008) y con duelo complicado (Golden, Dalgleish, \& Mackintosh, 2007) al ser preguntados por sus recuerdos autobiográficos muestran una menor especificidad en el recuerdo de los mismos, en condiciones específicas, en comparación con participantes que no muestran esta psicopatología. Sin embargo, otras investigaciones señalan que las memorias autobiográficas en el TEPT sí son coherentes y se encuentran bien integradas (Rubin, 2011).

La segunda aproximación sostiene que los recuerdos de hechos traumáticos pueden convertirse en algo central dentro de la memoria autobiográfica, puesto que se diferencian de las experiencias previas por su novedad y saliencia (Smeets, Giesbrecht, Raymaekers, Shaw, \& Merckelbach, 2010).
Dicha centralidad puede venir acompañada de consecuencias negativas para la persona, influyendo en su forma de procesar y dar significado a nuevas experiencias (Berntsen \& Rubin, 2006).

Para medir el grado en que una experiencia es central para el sujeto, Berntsen y Rubin (2006) desarrollaron la Centrality of Event Scale (CES), una escala compuesta por 20 ítems. Para su construcción parten de una definición tripartita, entendiendo que un evento es central cuando supone un punto de inflexión en la vida, cuando forma parte de la identidad y personalidad de la persona y cuando el acontecimiento se convierte en una referencia para atribuir significado a otras experiencias (Berntsen \& Rubin, 2006; Rubin, 2011). La centralidad de un evento está relacionada con las diferencias individuales que existen en el procesamiento de diferentes eventos traumáticos (Berntsen \& Rubin, 2011), y tendría como consecuencia un aumento en la accesibilidad y la intensidad de dichos recuerdos (Brown, Antonius, Kramer, Root, \& Hirst, 2010; Rubin, Berntsen, \& Bohni, 2008).

Diversas investigaciones han mostrado las relaciones existentes entre una alta centralidad y diferentes psicopatologías. Algunos ejemplos son la relación con la severidad del TEPT en niños con abuso sexual (Robinaugh \& McNally, 2011), en combatientes de guerra veteranos (Brown et al., 2010), en casos de dolor crónico (Perri \& Keefe, 2008) o en duelo complicado (Boelen, 2010, 2012). Así mismo, las puntuaciones en la CES se han relacionado con los valores de depresión y ansiedad, así como con diferentes estrategias de afrontamiento en varios estudios (Berntsen \& Rubin, 2006; Boals $\&$ Schuettler, 2011). En diseños cualitativos también aparece señalado el papel central que pueden ocupar recuerdos dolorosos o traumáticos, como es el caso de madres en proceso de duelo (Bosquet-del Moral et al., 2012) o en padres con hijos diagnosticados de parálisis cerebral infantil (FernándezAlcántara et al., 2013).

La centralidad de un evento traumático también se ha asociado con crecimiento postraumático, es decir, con la formulación de interpretaciones o puntos de vista adaptativos como resultado de haber 
vivenciado una experiencia traumática (Boals \& Schuettler, 2011). Así mismo, la CES se ha aplicado para medir la centralidad de eventos positivos, aunque en este caso las puntuaciones no muestran relación con sintomatología de estrés postraumático y estrés emocional (Berntsen, Rubin, \& Siegler, 2011). Se han encontrado diferencias de género (Boals, 2010) y en función de la edad (Boals, Hayslip, Knowles, \& Banks, 2012).

Desde un punto de vista psicométrico la CES ha mostrado una alta consistencia interna, con valores de alfa de Cronbach de 0.94 (Berntsen \& Rubin, 2006). Dichos autores obtuvieron evidencia a favor de una estructura unifactorial en la CES, aunque estudios con muestra clínica apuntan hacia la la necesidad de profundizar en el estudio de la estructura factorial de la misma (Robinaugh \& McCally, 2011).

Dada la utilidad clínica y las buenas propiedades psicométricas de la escala, el objetivo de esta investigación fue realizar la adaptación al castellano de la CES y obtener evidencias de fiabilidad y validez de la misma. Con respecto a esta última, se esperaba encontrar relaciones positivas de carácter moderado entre las puntuaciones de la CES y depresión, ansiedad y sintomatología de estrés postraumático.

\section{Método}

\section{Participantes}

Muestra 1. Estuvo compuesta por 208 estudiantes de primer curso de grado de la Universidad de Granada, de los cuales 46 fueron varones (22\%) y 162 mujeres (78\%). Con respecto al estado civil el $96 \%$ eran solteros y la media de edad fue de $20.73(D E=$ 5.8, Rango $=18-49$ ). Esta muestra se utilizó para el análisis de los ítems, la fiabilidad, y para obtener las evidencias de validez. Completaron todos los instrumentos que se detallan a continuación

Muestra 2. La segunda muestra estuvo formada por 320 estudiantes de la Universidad de Granada, de los cuales 88 fueron varones (27.5\%) y 232 mujeres (72.5\%). El $98 \%$ eran solteros y la media de edad fue de $20.5(D E=4.91$, Rango $=17-60)$.
Esta muestra se utilizó para el análisis de fiabilidad, así como para la estructura factorial de la escala. Completaron únicamente la CES

\section{Instrumentos}

\section{Centrality of Event Scale \\ (Berntsen Eु Rubin, 2006)}

Compuesta por 20 ítems retrotraducidos con un rango de respuesta entre 1 (completamente en desacuerdo) y 5 (completamente de acuerdo). Antes de completar la escala se pidió a los participantes que recordaran el suceso más traumático o estresante de su vida para completar la escala.

\section{Cuestionario de Ansiedad Estado Rasgo-STAI (Spielberg, Gorsuch, Ë Lushene, 1982)}

Incluye dos subescalas, denominadas STAI-Rasgo y STAI-Estado. Un reciente estudio (GuillénRiquelme \& Buela-Casal, 2011), evaluando sus propiedades psicométricas, ha mostrado una alta consistencia interna ( $\alpha=0.9$ para el rasgo y $\alpha=$ 0.94 para el estado). En esta muestra los valores de consistencia interna fueron de $\alpha=0.89$ para el estado y $\alpha=0.88$ para el rasgo.

Inventario de Depresión de Beck ([BDI]; Beck, Steer, $\mathcal{E}$ Carbin, 1988)

Mide la severidad de sintomatología depresiva con un total de 22 ítems. Se ha informado de un alfa de Cronbach de 0.89 y una validez concurrente de 0.41 (Ibañez, Peñate, \& González, 1997; Sanz \& Vázquez, 1998). En esta muestra los valores fueron de $\alpha=0.81$.

\section{Cuestionario de Estrés Post-Traumático ([PSS]; Foa, Riggs, Dancu, Eु Rothbaum, 1993).}

Se trata de una escala de 17 ítems elaborada a partir de los síntomas que caracterizan el trastorno de estrés postraumático. Ha mostrado una consistencia interna de $\alpha=0.85$ y una fiabilidad test-retest de 0.74 (Foa et al., 1993). Se utilizó la 
versión en español usada en otros estudios como el de Vera (2004) o el de Ferrando, Chico y Tous (2002). El alfa de Cronbach en esta muestra fue de $\alpha=0.84$.

\section{Procedimiento}

La escala fue retrotraducida del inglés al español por dos traductores independientes. En la versión en castellano se incluyó también un espacio para que los participantes escribiesen el tipo de acontecimiento en el que estaban pensando al contestar la escala. Las instrucciones fueron las siguientes: "Por favor recuerda el suceso más traumático o estresante de tu vida, escríbelo en el espacio correspondiente y contesta a las siguientes afirmaciones sinceramente, señalando en la escala del 1 al 5".

Con la primera versión de la escala se realizó un juicio de expertos contando con la colaboración de varios investigadores especialistas en procesos de fin de vida $(N=37)$ para comprobar la redacción de los ítems y las instrucciones, como se recomienda en los estándares (American Psychological Association [APA], American Educational Research Association [AERA] \& National Council on Measurement in Education [NCME], 1999; Muñiz, Elosua, \& Hambleton, 2013).

La primera muestra completó un cuadernillo formado por los instrumentos mencionados anteriormente (BDI, STAI, PSS y CES), que se volvieron a aplicar dos meses después y al año. Se utilizaron tres versiones contrabalanceadas del cuadernillo para evitar los efectos secuenciales. Dado que no se encontraron diferencias entre las secuencias, de aquí en adelante no se describen más. La segunda muestra completó solamente la CES. La aplicación de los cuestionarios en ambas muestras se realizó de manera grupal.

\section{Análisis de los Datos}

Para el análisis de los datos se empleó el programa estadístico SPSS (SPSS Inc., v 15.0) y el programa FACTOR (Lorenzo-Seva \& Ferrando, 2007). Se obtuvieron datos descriptivos de las puntuaciones de cada uno de los ítems para comprobar su com- portamiento estadístico en el análisis de ítems. La fiabilidad fue comprobada a través de la correlación entre el test y el retest, así como el alfa de Cronbach. Para examinar la estructura factorial de la escala se realizó un análisis de componentes principales. El número de factores extraídos se seleccionó a partir del gráfico de sedimentación, considerando, además el incremento de varianza explicada al añadir un nuevo factor y los resultados del análisis paralelo. Se realizaron correlaciones bivariadas de Pearson, con el objeto de obtener evidencias de validez a través de las relaciones de la CES con la depresión, la ansiedad y el estrés postraumático. Finalmente, se realizaron los análisis de regresión por pasos tomando como variable dependiente el estrés postraumático.

\section{Resultados}

\section{Análisis de los ítems}

En la Tabla 1 se muestran los descriptivos de los 20 ítems de la escala CES que compusieron la versión definitiva en castellano para la Muestra 1. Los ítems mostraron una distribución con valores medios en torno al punto medio de la escala. Todos los ítems muestran una desviación típica por encima de 1. Los valores de asimetría y curtosis se muestran en la Tabla 1 .

\section{Fiabilidad}

Las evidencias de fiabilidad de la escala se obtuvieron empleando una medida test-retest, así como el cálculo del alfa de Cronbach para la consistencia interna. Este último mostró una puntuación elevada para el total de los ítems tanto en la Muestra 1 ( $\alpha$ $=0.92)$, como en la Muestra $2(\alpha=0.94)$.

Para comprobar la estabilidad de las puntuaciones en el tiempo, se realizó un test-retest con una diferencia de 2 meses. Se seleccionaron aquellos participantes de la Muestra 1 en los que el evento utilizado para responder a la escala fue el mismo en la primera y la segunda medida, tomada a los dos meses. Esta muestra estuvo formada por 108 participantes. Los resultados mostraron una alta correlación $\left(r_{\text {test- }}\right.$ 
retest $=0.803, p<0.01)$ entre las puntuaciones totales. Así mismo, 27 de estos sujetos completaron la escala un año después, encontrándose también una correlación significativa $(r=0.558, p<0.01)$.

\section{Estructura factorial}

La estructura factorial de la escala se evaluó en la segunda muestra a través de un análisis de componentes principales con rotación VA-
RIMAX. El tamaño muestral era suficiente, de acuerdo con el criterio Kaiser-Meyer-Olkin $(\mathrm{KMO}=0.94)$ y el Test de Esfericidad de Bartlett $\left(\chi_{(190)}^{2}=3135.88, p<0.001\right)$. Los resultados muestran tres posibles factores con autovalores mayores que 1: 9.25, 1.21 y 1 , explicando el primero de ellos un $45.25 \%$ de la varianza y los dos siguientes un $6 \%$ y un $5 \%$, respectivamente. En el gráfico de sedimentación se observa una gran diferencia entre el primer factor y los demás, in-

TABLA 1

Análisis de los ítems en la Muestra 1

\begin{tabular}{|c|c|c|c|c|c|}
\hline Ítem & Media & $\mathrm{DE}$ & Asimetría & Curtosis & $\begin{array}{c}\text { Correlación } \\
\text { ítem-total } \\
\text { corregida }\end{array}$ \\
\hline $\begin{array}{l}\text { 1. Este suceso se ha convertido en un punto de referencia respecto a la } \\
\text { manera en que entiendo nuevas experiencias }\end{array}$ & 3.77 & 1.069 & -0.864 & 0.324 & 0.451 \\
\hline $\begin{array}{l}\text { 2. Automáticamente veo conexiones y similitudes entre el suceso y } \\
\text { experiencias actuales de mi vida }\end{array}$ & 3.02 & 1.183 & -0.19 & -0.818 & 0.429 \\
\hline 3. Siento que este suceso se ha convertido en parte de mi identidad & 3.32 & 1.191 & -0.397 & -0.725 & 0.613 \\
\hline $\begin{array}{l}\text { 4. Este suceso puede considerarse un referente o una marca de asuntos } \\
\text { importantes en mi vida }\end{array}$ & 3.53 & 1.048 & -0.668 & -0.001 & 0.558 \\
\hline 5. Este suceso está haciendo que mi vida sea diferente a la de los demás & 2.69 & 1.325 & 0.194 & -1.196 & 0.64 \\
\hline $\begin{array}{l}\text { 6. Este suceso ha afectado a mi manera de entenderme a mí mismo y al } \\
\text { mundo }\end{array}$ & 3.25 & 1.235 & -0.413 & -0.833 & 0.584 \\
\hline $\begin{array}{l}\text { 7. Creo que la gente que no ha experimentado este tipo de suceso pien- } \\
\text { sa de manera diferente a como lo hago yo }\end{array}$ & 3.47 & 1.176 & -0.493 & -0.655 & 0.466 \\
\hline 8. Este suceso dice mucho acerca de quién soy yo & 2.96 & 1.249 & -0.047 & -1.007 & 0.596 \\
\hline $\begin{array}{l}\text { 9. A menudo veo conexiones y similitudes entre este suceso y mis rela- } \\
\text { ciones actuales con los demás }\end{array}$ & 2.79 & 1.27 & 0.192 & -1.018 & 0.58 \\
\hline $\begin{array}{l}\text { 10. Siento que este suceso se ha convertido en una parte central en la } \\
\text { historia de mi vida }\end{array}$ & 3 & 1.295 & -0.082 & -1.104 & 0.646 \\
\hline $\begin{array}{l}\text { 11. Creo que la gente que no ha experimentado este tipo de suceso tie- } \\
\text { ne una manera de verse a sí misma distinta de la que yo tengo }\end{array}$ & 2.99 & 1.257 & -0.13 & -0.998 & 0.552 \\
\hline $\begin{array}{l}\text { 12. Este suceso ha influido en mi manera de concebir y sentir otras } \\
\text { experiencias }\end{array}$ & 3.64 & 0.914 & -0.733 & 0.45 & 0.552 \\
\hline $\begin{array}{l}\text { 13. Este suceso se ha convertido en un punto de referencia respecto a la } \\
\text { manera en que considero mi futuro }\end{array}$ & 3.07 & 1.244 & -0.114 & -0.984 & 0.538 \\
\hline $\begin{array}{l}\text { 14. Si tuviese que pensar en la historia de mi vida este suceso sería cen- } \\
\text { tral y estaría conectado a muchas otras experiencias }\end{array}$ & 2.86 & 1.25 & 0.018 & -1.01 & 0.609 \\
\hline $\begin{array}{l}\text { 15. Mi vida puede dividirse en dos capítulos principales: uno anterior y } \\
\text { otro posterior al suceso }\end{array}$ & 2.95 & 1.396 & 0.033 & -1.264 & 0.648 \\
\hline 16. Este suceso ha cambiado mi vida para siempre & 2.81 & 1.284 & 0.034 & -1.11 & 0.713 \\
\hline 17. Pienso en los efectos que éste suceso tendrá en mi futuro & 2.76 & 1.271 & 0.125 & -1.053 & 0.572 \\
\hline 18. Este suceso fue un punto de inflexión en mi vida & 3.38 & 1.178 & -0.519 & -0.566 & 0.654 \\
\hline 19. Si este evento no hubiese ocurrido sería una persona diferente & 3.34 & 1.304 & -0.342 & -1.005 & 0.62 \\
\hline 20. Cuando reflexiono sobre mi futuro a menudo recuerdo este suceso & 3.05 & 1.309 & -0.248 & -1.102 & 0.517 \\
\hline
\end{tabular}

Fuente: elaboración propia 


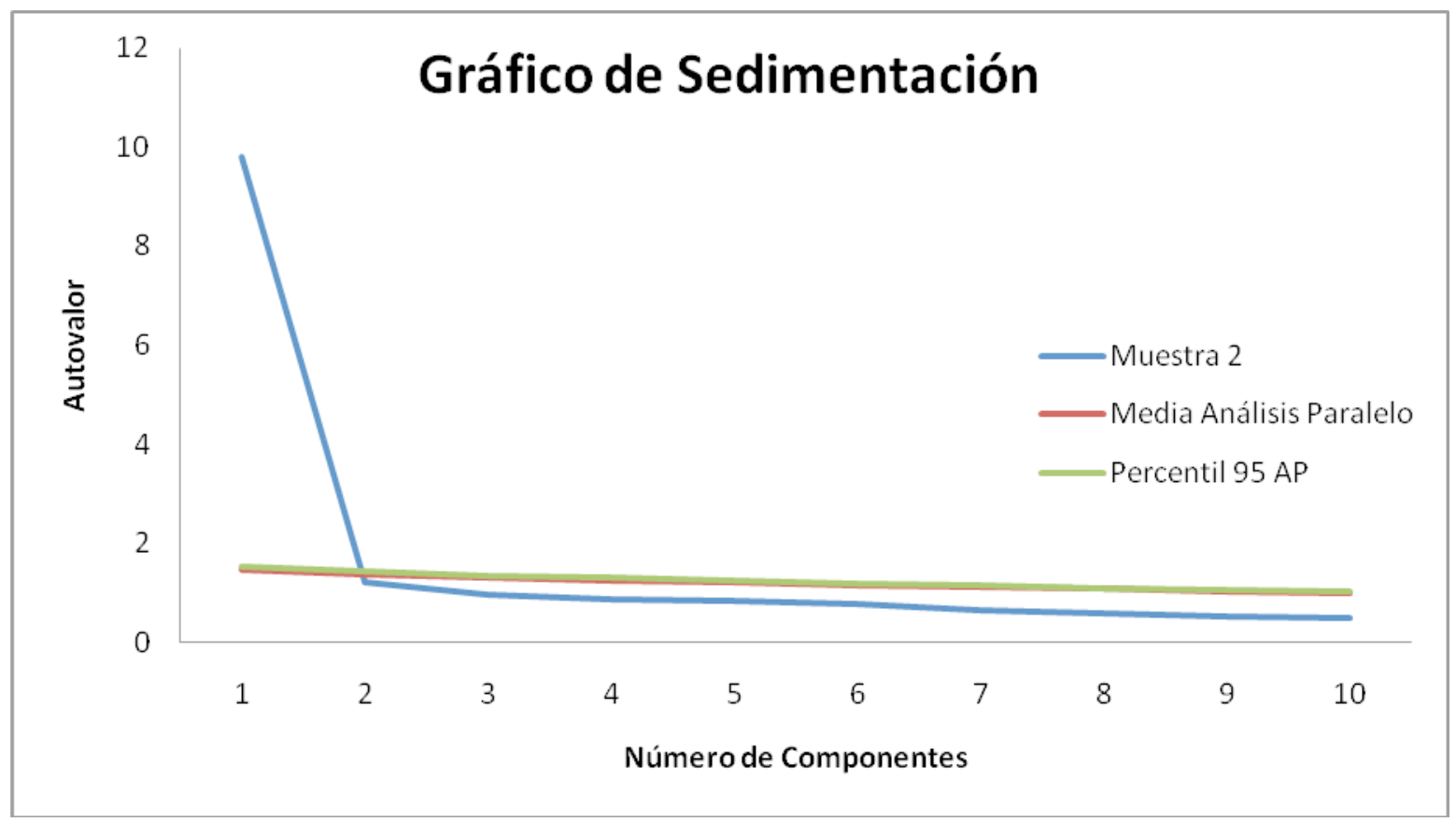

Figura 1. Gráfico de sedimentación de la Muestra 2 y de una muestra aleatoria con distribución normal (Análisis Paralelo).

Fuente: elaboración propia

dicando una solución unifactorial de agrupación de los ítems (Figura 1). Así mismo, se realizó un análisis paralelo (Hayton, Allen, \& Scarpello, 2004), siguiendo el método de Horn (1965). Para ello, se empleó la matriz de las correlaciones policóricas, al considerarse más adecuadas para este tipo de análisis. En la Figura 1 se muestra el gráfico de sedimentación de los datos originales junto con los resultados del análisis paralelo y del percentil 95 del mismo para los primeros 10 factores (Hayton et al., 2004). En la Tabla 2 se muestran los valores de las cargas factoriales para cada una de las tres variables extraídas a través del programa FACTOR (Lorenzo-Seva \&

TABLA 2

Autovalores obtenidos para los diferentes componentes a través del análisis de componentes principales y el análisis paralelo

\begin{tabular}{cccc}
\hline Componentes & Muestra 2 & Análisis Paralelo & Percentil 95 (AP) \\
\hline 1 & 9.79 & 1.47 & 1.55 \\
2 & 1.21 & 1.38 & 1.44 \\
3 & 0.97 & 1.32 & 1.36 \\
4 & 0.88 & 1.26 & 1.31 \\
5 & 0.83 & 1.21 & 1.25 \\
6 & 0.79 & 1.16 & 1.2 \\
7 & 0.64 & 1.12 & 1.16 \\
8 & 0.58 & 1.08 & 1.11 \\
9 & 0.52 & 1.04 & 1.07 \\
10 & 0.49 & 1 & 1.03 \\
\hline
\end{tabular}

Fuente: elaboración propia 
TABLA 3

Correlaciones bivariadas entre los constructos en Muestra 1

\begin{tabular}{lccccc}
\hline & CES & BDI & PSS & STAI-E & STAI-R \\
\hline CES & 1 & $0.324(* *)$ & $0.326(* *)$ & $0.279(* *)$ & $0.304(* *)$ \\
BDI & & 1 & $0.472(* *)$ & $0.577(* *)$ & $0.654(* *)$ \\
PSS & & 1 & $0.447(* *)$ & $0.553(* *)$ \\
STAI-E & & & 1 & $0.689(* *)$ \\
STAI-R & & & & & 1 \\
\hline
\end{tabular}

** La correlación es significativa $(p<0.01)$.

Fuente: elaboración propia

Ferrando, 2007). Los resultados parecen indicar una estructura unifactorial de la escala.

\section{Validez}

Las evidencias de validez concurrente se obtuvieron a través del coeficiente de correlación de Pearson entre las puntuaciones de los diferentes instrumentos, en la primera muestra. Las correlaciones muestran una relación positiva y significativa entre centralidad del evento y ansiedad estado, ansiedad rasgo, depresión y sintomatología de estrés postraumático (Tabla 3).

La muestra de participantes se dividió en dos grupos a partir de sus puntuaciones totales en TEPT. Para ello seleccionamos los percentiles 27 (puntuación menor o igual a 8) y 73 (puntuación mayor o igual a 18). Las puntuaciones totales en la escala CES de estos grupos se sometieron a un
ANOVA unifactorial que indicó diferencias significativas entre ellos $F(1,115)=13.529, p<0.001$, sugiriendo que la escala discrimina correctamente entre ambos grupos.

Para comprobar si la centralidad del evento era predictora de la sintomatología de estrés postraumático, se llevó a cabo un análisis de regresión múltiple por pasos introduciendo como predictores las puntuaciones de las escalas CES, BDI y STAI-R (Tabla 4). El mejor modelo (32\% de varianza explicada) incluyó las tres variables.

\section{Discusión}

El objetivo de este estudio fue adaptar al castellano la Centrality of Event Scale, y determinar su validez y fiabilidad. Esta escala ha demostrado ser útil para evaluar aspectos directamente relacionados con el procesamiento de eventos traumáticos (Berntsen \&

TABLA 4

Modelo de regresión tomando como variable dependiente el estrés postraumático

\begin{tabular}{ccccccc}
\hline Modelo & & Beta Estandarizada & $t$ & Sig. & R2Corregida & Cambio en R2 \\
\hline 1 & (Constante) & & 1.281 & 0.202 & & \\
& CES_Total & 0.317 & 4.490 & 0 & 0.096 & 20.159 \\
& (Constante) & & 1.639 & 0.103 & & \\
& CES_Total & 0.179 & 2.639 & 0.009 & & \multirow{2}{*}{0.252} \\
& BDI_Total & 0.423 & 6.215 & 0 & & \\
& (Constante) & & 0.089 & 0.929 & & \\
& CES_Total & 0.134 & 2.054 & 0.041 & & \\
& BDI_Total & 0.198 & 2.456 & 0.015 & & \\
& STAI_Rasgo & 0.372 & 4.625 & 0 & 0.329 & \\
\hline
\end{tabular}

Fuente: elaboración propia 
Rubin, 2011). El análisis de ítems señala que éstos cumplen unas adecuadas propiedades psicométricas, siendo éste el primer estudio en reportarlas. Los ítems presentan valores ligeramente superiores al valor medio de la Escala, algo esperable puesto que se les pedía a los participantes que pensaran en el mayor evento traumático que hubiesen vivido. En todos se encuentra una variabilidad de respuestas aceptable.

Con respecto a la fiabilidad de la escala el alfa de Cronbach encontrado coincide con el de otros estudios (Berntsen et al., 2011; Boals, 2010). Así mismo, las puntuaciones parecen ser estables, tanto a los dos meses como en una posterior medida longitudinal al año.

En relación a las evidencias de validez, el análisis de componentes principales parece indicar que la escala es unifactorial, lo que concuerda con lo encontrado inicialmente por Berntsen y Rubin (2006). Ahora bien, en su trabajo original estos autores plantean que los ítems fueron creados a partir de tres aspectos característicos de la centralidad: que el evento afecte a la identidad, que sea un punto de inflexión y que influya en las interpretaciones de futuras experiencias (Berntsen \& Rubin, 2006). Los resultados de la adaptación al castellano, al igual que la versión original, no han corroborado estos tres componentes de la centralidad, lo que sugiere la necesidad de indagar más específicamente en la definición semántica del constructo en futuras investigaciones.

Al desarrollar un instrumento de medida se asume que los ítems son indicadores del constructo que previamente ha sido definido, los componentes que se incluyen en la definición deben estar representados por los ítems que se desarrollan (De los Santos-Roig \& Pérez-Meléndez, 2013). En la adaptación al castellano los ítems funcionan en una única dirección, midiendo centralidad del evento, sin hacer distinciones entre los tres componentes conceptuales de la misma. Aún así, en los diferentes estudios que utilizan la CES (Berntsen \& Rubin, 2006; Berntsen et al., 2011; Boals \& Schuettler, 2011) no se especifica qué papel diferencial, si es que lo hubiera, puede tener cada uno de estos com- ponentes en la predicción de la sintomatología de TEPT ni de otros trastornos.

Las correlaciones entre las puntuaciones de la CES con otros constructos coinciden con los resultados de otras investigaciones, en las que también se han observado relaciones positivas con depresión, ansiedad y TEPT (Berntsen \& Rubin, 2006; Berntsen et al., 2011). Así mismo, el análisis de regresión ha mostrado que la CES predice significativamente las puntuaciones en sintomatología de TEPT, tanto considerada aisladamente como con otras variables importantes, resultados que van en la línea de diversas investigaciones (Boals et al., 2012; Boals \& Schuettler, 2011). Futuros estudios deben ir orientados hacia conocer qué otras variables se relacionan con la centralidad del evento. Conocer cómo se comporta la escala en población diagnosticada de TEPT (Botero-García, 2004) o de duelo complicado (Boelen, 2012) es un objetivo que no debe perderse de vista, ampliando de este modo el alcance y la utilidad práctica de la misma.

Como principal limitación de este trabajo se podría señalar que la adaptación se ha realizado en una muestra compuesta por estudiantes universitarios, al igual que en la escala original. Esto, a pesar de las críticas históricas al uso de estudiantes en la investigación (Peterson, 2001), tiene su razón de ser, al margen de cuestiones puramente pragmáticas o de accesibilidad. El comienzo de los estudios universitarios es un momento importante para la vida de las personas, cuando puede tener lugar un gran número de sucesos emocionalmente estresantes y traumáticos. Bados, Greco y Toribio (2012) encontraron que el $60.6 \%$ de una muestra de 432 universitarios habían experimentado algún tipo de acontecimiento traumático, siendo los más frecuentes la muerte de algún ser querido, delitos, atracos o accidentes de tráfico. En un estudio longitudinal realizado con estudiantes universitarios Galatzer-Levy, Burton y Bonanno (2012) encontraron que el $50.6 \%$ de los estudiantes habían vivenciado un evento traumático en su primer año, y que los patrones de respuesta al estrés entre individuos eran diferentes, mostrando la influencia de variables como la ansiedad en el apego, la ego-resiliency (Galatzer-Levy \& Bonanno, 2012) y la flexibilidad 
en el afrontamiento (Bonanno, Pat-Horenczyk, \& Noll, 2011; Galatzer-Levy \& Bonanno, 2012). De ahí que se considerara de interés utilizar este tipo de muestra para la adaptación de la escala.

En conclusión, tanto la validez como la fiabilidad de la adaptación española de la CES indican que esta escala puede utilizarse en investigaciones en las que la centralidad del evento sea una variable importante. Futuras investigaciones deberán dirigirse a estudiar el comportamiento de esta escala en muestras clínicas, para comprobar el papel predictor de la centralidad en diferentes trastornos.

\section{Referencias}

American Psychological Association, American Educational Research Association \& National Council on Measurement in Education. (1999). Standards for educational and psychological tests. Washington, DC: Autores.

Bados, A., Greco, A., \& Toribio, L. (2012). Experiencias traumáticas y trastorno por estrés postraumático en universitarios españoles. Anales de Psicología, 28(2), 387-396.

Beck, A.T., Steer, R. A., \& Carbin, M. G. (1988). Psychometric properties of the Beck Depression Inventory: Twenty-five years of evaluation. Clinical Psychology Review, 8, 77-100.

Berntsen, D., \& Rubin, D. C. (2006). The Centrality of Events Scale: A measure of integrating a trauma into one's identity and its relation to post-traumatic stress disorder symptoms. Behavior Research and Therapy, 44(2), 219-231.

Berntsen, D., Rubin, D. C., \& Siegler, I. C. (2011). Two versions of life: Emotionally negative and positive life events have different roles in the organization of life story and identity. Emotion, 11(5), 1190-1201.

Boals, A. (2010). Autobiographical memories that have become central to identity: Gender differences in the Centrality of Events Scale for positive and negative events. Applied Cognitive Psychology, 24(1), 107-121. doi: 10.1002/acp.1548

Boals, A., Hayslip, B., Knowles, L., \& Banks, J. B. (2012). Perceiving a negative event as central to one's identity partially mediates age differences in PTSD symptoms. Journal of Aging and Health, 24(3), 459-474.

Boals, A., \& Schuettler, D. (2011). A double-edged sword: Event centrality, PTSD and post-traumatic growth. Applied Cognitive Psychology, 25(5), 817822.

Boelen, P. A. (2012). A prospective examination of the association between the centrality of a loss and post-loss psychopathology. Journal of Affective Disorders, 137(1-3), 117-124.

Bonanno, G. A., Pat-Horenzcyk, R., \& Noll, J. E. (2011). Coping flexibility and trauma: The Perceived Ability to Cope with Trauma (PACT) Scale. Psychological Trauma, 3(2), 117-129.

Bosquet-del Moral, L., Campos-Calderón, C. P., HuesoMontoro, C., Pérez-Marfil, N., Hernández-Molinero, Á., Arcos-Ocón, L., \& Cruz-Quintana, F. (2012). Vivencias y experiencias de duelo en madres tras un proceso de enfermedad oncológica de sus hijos. Medina Paliativa, 19(2), 64-72.

Botero-García, C. (2005). Efectividad de una intervención cognitivo-conductual para el trastorno de estrés post-traumático en excombatientes colombianos. Universitas Psycologica, 4(2), 205-219.

Brown, A. D., Antonius, D., Kramer, M., Root, J. C., \& Hirst, W. (2010). Trauma centrality and PTSD in veterans returning from Iraq and Afghanistan. Journal of Traumatic Stress, 23(4), 496-499.

Dalgleish, T. (2004). Cognitive theories of posttraumatic stress disorder: The evolution of multi-representational theorizing. Psychological Bulletin, 130(2), 228-260.

Dalgleish, T., Rolfe, J., Golden, A. M., Dunn, B. D., \& Barnard, P. J. (2008). Reduced autobiographical memory specificity and posttraumatic stress: Exploring the contributions of impaired executive control and affect regulation. Journal of Abnormal Psychology, 117(1), 236-41.

De los Santos-Roig, M., \& Pérez-Meléndez, C. (2013). De la definición semántica a los ítems a través de las tablas de especificaciones: elaboración de la escala sobre representación de la enfermedad, E.R.C.E. Anales de Psicología, 29(2), 345-359.

Dekel, S., \& Bonanno, G.A. (2013). Changes in trauma memory and patterns of posttraumatic stress. Psy- 
chological Trauma: Theory, Research, Practice, and Policy, 5(1), 26-34.

Fernández-Alcántara, M., García-Caro, M. P., BerrocalCastellano, M., Benítez-Feliponi, Á, Robles-Vizcaíno, C., \& Laynez-Rubio, C. (2013). Experiencias y cambios en los padres de niños con parálisis cerebral infantil: estudio cualitativo. Anales del Sistema Sanitario de Navarra, 36(1), 9-20

Ferrando, P. J., Chico, E., \& Tous, J. M. (2002). Propiedades psicométricas del test de optimismo Life Orientation Test. Psicothema, 14(3), 673-680.

Foa, B. E., Riggs, D. S., Dancu, C. V., \& Rothbaum, B. O. (1993). Realibility and validity of a brief instrument for assessing posttraumatic stress disorder. Journal of Traumatic Stress, 6, 459-473.

Galatzer-Levy, I., Burton, C. L., \& Bonanno, G. A. (2012). Coping flexibility, potentially traumatic life events, and resilience: A prospective study of college student adjustment. Journal of Social and Clinical Psychology, 31(6), 542-567.

Galatzer-Levy, I. R., \& Bonanno, G. A. (2012). Heterogeneous patterns of stress over the four years of college: Associations with anxious attachment and ego-resiliency. Journal of Personality. Publicación anticipada en línea. doi: 10.1111/jopy.12010

Golden, M. A., Dalgleish, T., \& Mackintosh, B. (2007). Levels of specificity of autobiographical memories and of biographical memories of the deceased in bereaved individuals with and without complicated grief. Journal of Abnormal Psychology, 116(1), 786-795.

Guillén-Riquelme, A., \& Buela-Casal, G. (2011). Actualización psicométrica y funcionamiento diferencial de los ítems en el State Trait Anxiety Inventory (STAI). Psicothema, 23(3), 510-515.

Hayton, J. C., Allen, D .G., \& Scarpello, V. (2004). Factor retention decisions in exploratory factor analysis: A tutorial on Parallel Analysis. Organizational Research Methods, 7(2), 191-205.

Horn, J. L. (1965). A rationale and test for the number of factors in factor analysis. Psychometrika, 30, 179-185.

Ibáñez, I., Peñate, W., \& González, M. (1997). La estructura factorial del Inventario de Depresión de Beck. Psicología Conductual, 5, 71-91.
Klein, S. B., German, T. P., Cosmides, L., \& Gabriel, R. (2004). A theory of autobiographical memory: Necessary components and disorders resulting from their loss. Social Cognition, 22(5), 460-490.

Lorenzo-Seva, U., \& Ferrando, P. (2007). FACTOR: A computer program to fit the exploratory factor analysis model. Tarragona: University Rovira y Virgili.

Moreno, B., Morante, M. E., Rodríguez, R., \& Rodríguez, A. (2008). Resistencia y vulnerabilidad ante el trauma: el efecto moderador de las variables de personalidad. Psicothema, 20(1), 124-130.

Muñiz, J., Elosua, P., \& Hambleton, R. K. (2013). Directrices para la traducción y adaptación de los test (2. - ed.). Psicothema, 25(2), 151-157.

Perri, L. M., \& Keefe, F. J. (2008). Applying centrality of event to persistent pain: A preliminary view. Journal of Pain, 9(3), 265-271.

Peterson, R. A. (2001). On the use of college students in social science research: Insights from a second order meta-analysis. Journal of Consumer Research, 28(3), 450-461.

Robinaugh, D. J., \& McNally, R. J. (2011). Trauma centrality and PTSD symptom severity in adult survivors of childhood sexual abuse. Journal of Traumatic Stress, 24(4), 483-486.

Rubin, D. C. (2011). The coherence of memories for trauma: Evidence from posttraumatic stress disorder. Consciousness and Cognition, 20(3), 857-865.

Rubin, D. C., Berntsen, D., \& Bohni, M. K. (2008). A memory-based model of posttraumatic stress disorder: Evaluating basic assumptions underlying the PTSD diagnosis. Psychological Review, 115(4), 985-1011.

Sanz, J., \& Vázquez, C. (1998). Fiabilidad, validez y datos normativos del inventario para la depresión de Beck. Psicothema, 10(2), 303-318.

Smeets, T., Giesbrecht, T., Raymaekers, L., Shaw, J., \& Merckelbach, H. (2010). Autobiographical integration of trauma memories and repressive coping predict post-traumatic stress symptoms in undergraduate students. Clinical Psychology $\mathbb{E}$ Psychotherapy, 17(3), 211-218.

Spielberger, C. D., Gorsuch, R. L., \& Lushene, R. (1982). Manual del Cuestionario de Ansiedad Estado/Rasgo (STAI). Madrid: Ediciones TEA. 
Vera, N. (2004). Tratamiento cognitivo-conductual del trastorno de estrés post-traumático crónico en una víctima de abusos sexuales en la infancia. Avances en Psicología Latinoamericana, 22, 89-103. 
